\title{
Existence results for impulsive neutral stochastic functional integro-differential inclusions with infinite delays
}

\author{
Jong Yeoul Park' and Jae Ug Jeong ${ }^{2^{*}}$
}

\begin{tabular}{l}
\hline${ }^{*}$ Correspondence: \\
jujeong@deu.ac.kr \\
2Department of Mathematics, \\
Dongeui University, Busan, 614-714, \\
South Korea \\
Full list of author information is \\
available at the end of the article
\end{tabular}

available at the end of the article

\begin{abstract}
In this paper, we prove the existence of mild solutions for a class of impulsive neutral stochastic functional integro-differential inclusions with infinite delays in Hilbert spaces. The results are obtained by using the fixed-point theorem for multi-valued operators due to Dhage. An example is provided to illustrate the theory.

MSC: 93B05; 93E03
\end{abstract}

Keywords: impulsive equation; stochastic functional inclusion; mild solution; infinite delay

\section{Introduction}

In this paper, we shall consider the existence of mild solutions for impulsive neutral stochastic functional integro-differential inclusions with infinite delay of the following form:

$$
\begin{aligned}
& d\left[x(t)-g\left(t, x_{t}, \int_{0}^{t} a\left(t, s, x_{s}\right) d s\right)\right] d t \\
& \quad \in\left[A x(t)+f\left(t, x_{t}\right)\right] d t+F\left(t, x_{t}\right) d w(t), \quad t \in J=[0, b], t \neq t_{k}, \\
& \Delta x\left(t_{k}\right)=x\left(t_{k}^{+}\right)-x\left(t_{k}^{-}\right)=I_{k}\left(x\left(t_{k}^{-}\right)\right), \quad k=1,2, \ldots, m, \\
& x(t)=\phi(t) \in L^{2}\left(\Omega, \mathcal{B}_{h}\right) \quad \text { for a.e. } t \in J_{0}=(-\infty, 0],
\end{aligned}
$$

where the state $x(\cdot)$ takes values in a separable real Hilbert space $H$ with inner product $(\cdot, \cdot)$ and norm $|\cdot|, A$ is the infinitesimal generator of a compact analytic resolvent operator $S(t)$, $t \geq 0$, in the Hilbert space $H$. Suppose that $\{w(t): t \geq 0\}$ is a given $K$-valued Brownian motion or Wiener process with a finite trace nuclear covariance operator $Q \geq 0$ and $L(K, H)$ denotes the space of all bounded linear operators from $K$ into $H$. Further $a: D \times \mathcal{B}_{h} \rightarrow H$, $g: J \times \mathcal{B}_{h} \times H \rightarrow H, f: J \times \mathcal{B}_{h} \rightarrow H$ and $F: J \times \mathcal{B}_{h} \rightarrow \mathcal{P}\left(L_{Q}(K, H)\right)$ are given functions, where $D=\{(t, s) \in J \times J: s \leq t\}, \mathcal{P}\left(L_{Q}(K, H)\right)$ is the family of all nonempty subsets of $L_{Q}(K, H)$ and $L_{Q}(K, H)$ denotes the space of all Q-Hilbert-Schmidt operators from $K$ into $H$, which will be defined in Section 2. Here, $I_{k} \in C(H, H)(k=1,2, \ldots, m)$ are bounded functions. Furthermore, the fixed times $t_{k}$ satisfies $0=t_{0}<t_{1}<t_{2}<\cdots<t_{m}<b, x\left(t_{k}^{+}\right)$and $x\left(t_{k}^{-}\right)$denote the right and left limits of $x(t)$ at $t=t_{k} . \Delta x\left(t_{k}\right)=x\left(t_{k}^{+}\right)-x\left(t_{k}^{-}\right)=I_{k}\left(x\left(t_{k}^{-}\right)\right)$represents the jump in the state $x$ at time $t_{k}$, where $I_{k}$ determines the size of jump. The his- 
tories $x_{t}: \Omega \rightarrow \mathcal{B}_{h}, t \geq 0$, which are defined by setting $x_{t}=\{x(t+s): s \in(-\infty, 0]\}$, belong to the abstract phase space $\mathcal{B}_{h}$, which will be defined in Section 2 . The initial data $\phi=\{\phi(t):-\infty<t \leq 0\}$ is an $\mathcal{F}_{0}$-measurable, $\mathcal{B}_{h}$-valued random variables independent of $\{w(t): t \geq 0\}$ with finite second moment.

The theory of impulsive integro-differential inclusions has become an active area of investigation due to their applications in the fields such as mechanics, electrical engineering, medicine biology, ecology, and so on (see $[1,2]$ and references therein).

The existence of impulsive neutral stochastic functional integro-differential equations or inclusions with infinite delays have attracted great interest of researchers. For example, $\mathrm{Lin}$ and $\mathrm{Hu}$ [3] consider the existence results for impulsive neutral stochastic functional integro-differential inclusions with nonlocal initial conditions. Hu and Ren [4] studied the existence results for impulsive neutral stochastic functional integro-differential equations with infinite delays.

Motivated by the previous mentioned papers, we prove the existence of solutions for impulsive neutral stochastic functional integro-differential inclusions with infinite delays.

\section{Preliminaries}

Throughout this paper, $(H,|\cdot|)$ and $\left(K,|\cdot|_{K}\right)$ denote two real separable Hilbert spaces. Let $(\Omega, \mathcal{F}, P ; F)\left(F=\left\{\mathcal{F}_{t}\right\}_{t \geq 0}\right)$ be a complete filtered probability space satisfying the requirement that $\mathcal{F}_{0}$ contains all $P$-null sets of $\mathcal{F}$. An $H$-valued random variable is an $\mathcal{F}$-measurable function $x(t): \Omega \rightarrow H$ and the collection of random variables $S=\{x(t, w)$ : $\Omega \rightarrow H \mid t \in J\}$ is called a stochastic process. Suppose that $\{w(t): t \geq 0\}$ is a cylindrical $K$-valued Wiener process with a finite trace nuclear covariance operator $Q \geq 0$, denote $T_{r} Q=\sum_{i=1}^{\infty} \lambda_{i}=\lambda<\infty$, which satisfies $Q e_{i}=\lambda_{i} e_{i}$. So, actually, $w(t)=\sum_{i=1}^{\infty} \sqrt{\lambda_{i}} w_{i}(t) e_{i}$, where $\left\{w_{i}(t)\right\}_{i=1}^{\infty}$ are mutually independent one-dimensional standard Wiener process. We assume that $\mathcal{F}_{t}=\sigma\{w(s): 0 \leq s \leq t\}$ is the $\sigma$-algebra generated by $w$ and $\mathcal{F}_{T}=\mathcal{F}$. Let $\psi \in L(K, H)$ and define

$$
|\psi|_{Q}^{2}=T_{r}\left(\psi Q \psi^{*}\right)=\sum_{n=1}^{\infty}\left|\sqrt{\lambda_{n}} \psi e_{n}\right|^{2} .
$$

If $|\psi|_{Q}<\infty$, then $\psi$ is called a $Q$-Hilbert-Schmidt operator. Let $L_{Q}(K, H)$ denote the space of all Q-Hilbert-Schmidt operator $\psi: K \rightarrow H$. The completion $L_{Q}(K, H)$ of $L(K, H)$ with respect to the topology induced by the norm $|\cdot|_{Q}$, where $|\psi|_{Q}^{2}=\langle\psi, \psi\rangle$ is a Hilbert space with the above norm topology.

Let $A: D(A) \rightarrow H$ be the infinitesimal generator of a compact, analytic resolvent operator $S(t), t \geq 0$. Let $0 \in \rho(A)$. Then it is possible to define the fractional power $(-A)^{\alpha}$ for $0<\alpha \leq 1$ as a closed linear operator with its domain $D\left((-A)^{\alpha}\right)$ being dense in $H$. We denote by $H_{\alpha}$ the Banach space $D\left(-A^{\alpha}\right)$ endowed with the norm $\|x\|_{\alpha}=\left\|(-A)^{\alpha} x\right\|$, which is equivalent to the graph norm of $(-A)^{\alpha}$.

Lemma 2.1 ([5]) The following properties hold:

(i) If $0<\beta<\alpha \leq 1$, the $H_{\alpha} \subset H_{\beta}$ and the embedding is continuous and compact whenever the resolvent operator of $A$ is compact.

(ii) For every $0<\alpha<1$, there exists a positive constant $c_{\alpha}$ such that

$$
\left\|(-A)^{\alpha} S(t)\right\| \leq \frac{C_{\alpha}}{t^{\alpha}}, \quad t>0
$$


Now, we define the abstract phase space $\mathcal{B}_{h}$. Assume that $h:(-\infty, 0] \rightarrow(0, \infty)$ is a continuous function with $l=\int_{-\infty}^{0} h(t) d t<\infty$. For any $a>0$ we define

$$
\begin{gathered}
\mathcal{B}_{h}=\left\{\psi:(-\infty, 0] \rightarrow H:\left(E|\psi(\theta)|^{2}\right)^{\frac{1}{2}}\right. \text { is a bounded and measurable } \\
\text { function on } \left.[-a, 0] \text { and } \int_{-\infty}^{0} h(s) \sup _{s \leq \theta \leq 0}\left(E|\psi(\theta)|^{2}\right)^{\frac{1}{2}} d s<\infty\right\} .
\end{gathered}
$$

If $\mathcal{B}_{h}$ is endowed with the norm

$$
\|\psi\|_{\mathcal{B}_{h}}=\int_{-\infty}^{0} h(s) \sup _{s \leq \theta \leq 0}\left(E|\psi(\theta)|^{2}\right)^{\frac{1}{2}} d s \quad \text { for all } \psi \in \mathcal{B}_{h},
$$

then $\left(\mathcal{B}_{h},\|\cdot\|_{\mathcal{B}_{h}}\right)$ is a Banach space [6]. Now, we consider the space

$$
\begin{aligned}
\mathcal{B}_{b}= & \left\{x:(-\infty, b] \rightarrow H \text { such that } x_{k} \in C\left(J_{k}, H\right)\right. \text { and there exist } \\
& x\left(t_{k}^{+}\right) \text {and } x\left(t_{k}^{-}\right) \text {with } x\left(t_{k}\right)=x\left(t_{k}^{-}\right), x_{0}=\phi \in L^{2}\left(\Omega, \mathcal{B}_{h}\right) \text { on } \\
& (-\infty, 0], k=1,2, \ldots, m\},
\end{aligned}
$$

where $x_{k}$ is the restriction of $x$ to $J_{k}=\left(t_{k}, t_{k+1}\right], k=0,1, \ldots$, m. Let $\|\cdot\|_{b}$ be a seminorm in $\mathcal{B}_{b}$ defined by

$$
\|x\|_{b}=\left\|x_{0}\right\|_{\mathcal{B}_{h}}+\sup _{0 \leq s \leq b}\left(E|x(s)|^{2}\right)^{\frac{1}{2}}, \quad x \in \mathcal{B}_{b}
$$

Lemma 2.2 ([7]) Assume that $x \in \mathcal{B}_{b}$, then for $t \in J, x_{t} \in \mathcal{B}_{h}$. Moreover

$$
l\left(E|x(t)|^{2}\right)^{\frac{1}{2}} \leq\left\|x_{t}\right\|_{\mathcal{B}_{h}} \leq\left\|x_{0}\right\|_{\mathcal{B}_{h}}+l \sup _{0 \leq s \leq t}\left(E|x(t)|^{2}\right)^{\frac{1}{2}},
$$

where $l=\int_{-\infty}^{0} h(s) d s<\infty$.

We use the notation $\mathcal{P}(H)$ for the family of all subsets $H$ and denote

$$
\begin{aligned}
& \mathcal{P}_{c l}(H)=\{Y \in \mathcal{P}(H): Y \text { is closed }\}, \\
& \mathcal{P}_{b d}(H)=\{Y \in \mathcal{P}(H): Y \text { is bounded }\}, \\
& \mathcal{P}_{c v}(H)=\{Y \in \mathcal{P}(H): Y \text { is convex }\}, \\
& \mathcal{P}_{c p}(H)=\{Y \in \mathcal{P}(H): Y \text { is compact }\} .
\end{aligned}
$$

A multi-valued mapping $\Gamma: H \rightarrow \mathcal{P}(H)$ is called upper semicontinuous (u.s.c) if for any $x \in H$, the set $\Gamma(x)$ is a nonempty closed subset of $H$ and if for each open set $G$ of $H$ containing $\Gamma(x)$, there exists an open neighborhood $N$ of $x$ such that $\Gamma(N) \subseteq G$. $\Gamma$ is said to be completely continuous if $\Gamma(B)$ is relatively compact for every bounded subset of $B \subseteq H$. If the multi-valued mapping $\Gamma$ is completely continuous with nonempty compact values, then $\Gamma$ is u.s.c. if and only if $\Gamma$ has a closed graph, i.e., $x_{n} \rightarrow x, y_{n} \rightarrow y, y_{n} \in \Gamma\left(x_{n}\right)$ imply $y \in \Gamma(x)$. 
Definition 2.1 The multi-valued mapping $F: J \times \mathcal{B}_{h} \rightarrow \mathcal{P}(H)$ is said to be $L^{2}$-Carathéodory if

(i) $t \mapsto F(t, v)$ is measurable for each $v \in \mathcal{B}_{h}$,

(ii) $v \mapsto F(t, v)$ is u.s.c. for almost all $t \in J$ and $v \in \mathcal{B}_{h}$,

(iii) for each $q>0$, there exists $h_{q} \in L^{1}\left(J, \mathbb{R}^{+}\right)$such that

$$
\|F(t, v)\|^{2}=\sup _{f \in F(t, v)} E\left(|f|^{2}\right) \leq h_{q}(t)
$$

for all $\|v\|_{\mathcal{B}_{h}}^{2} \leq q$ and for a.e. $t \in J$.

The following lemma is crucial in the proof of our main result.

Lemma 2.3 ([8]) Let I be a compact interval and $H$ be a Hilbert space. Let $F$ be an $L^{2}$ Carathéodory multi-valued mapping with $N_{F, x} \neq \phi$ and let $\Gamma$ be a linear continuous mapping from $L^{2}(I, H)$ to $C(I, H)$. Then the operator

$$
\Gamma \circ N_{F}: C(I, H) \rightarrow \mathcal{P}_{c p, c v}(H), \quad x \mapsto\left(\Gamma \circ N_{F}\right)(x)=\Gamma\left(N_{F, x}\right)
$$

is a closed graph operator in $C(I, H) \times C(I, H)$, where $N_{F, x}$ is known as the selectors set from $F$; it is given by

$$
\sigma \in N_{F, x}=\left\{\sigma \in L^{2}(L(K, H)): \sigma(t) \in F(t, x) \text { for a.e. } t \in J\right\} .
$$

Theorem 2.1 ([9]) Let $X$ be a Banach space, $\Phi_{1}: X \rightarrow \mathcal{P}_{c l, c v, b d}(X)$ and $\Phi_{2}: X \rightarrow \mathcal{P}_{c p, c v}(X)$ be two multi-valued operators satisfying:

(a) $\Phi_{1}$ is a contraction,

(b) $\Phi_{2}$ is u.s.c. and completely continuous.

Then either

(i) the operator inclusion $\lambda x \in \Phi_{1} x+\Phi_{2} x$ has a solution for $\lambda=1$, or

(ii) the set $G=\left\{x \in X: \lambda x \in \Phi_{1} x+\Phi_{2} x, \lambda>1\right\}$ is unbounded.

Lemma 2.4 ([10]) Let $v, w:[0, b] \rightarrow[0, \infty)$ be continuous functions. If $w$ is nondecreasing and there are constants $\theta>0,0<\alpha<1$ such that

$$
v(t) \leq w(t)+\theta \int_{0}^{t} \frac{v(s)}{(t-s)^{1-\alpha}} d s, \quad t \in J
$$

then

$$
v(t) \leq e^{\frac{\theta^{n} \Gamma(\alpha)^{n} t^{n \alpha}}{\Gamma(n \alpha)}} \sum_{j=0}^{n-1}\left(\frac{\theta b^{\alpha}}{\alpha}\right)^{j} w(t)
$$

for every $t \in J$ and every $n \in N$ such that $n \alpha>1$ and $\Gamma(\cdot)$ is the Gamma function.

\section{Main result}

Let $J_{1}=(-\infty, b]$. First, we present the definition of the mild solution of problem (1.1)-(1.3).

Definition 3.1 A stochastic process $x: J_{1} \times \Omega \rightarrow H$ is called a mild solution of problem (1.1)-(1.3) if 
(i) $x(t)$ is measurable and $\mathcal{F}_{t}$-adapted for each $t \geq 0$,

(ii) $\Delta x\left(t_{k}\right)=x\left(t_{k}^{+}\right)-x\left(t_{k}^{-}\right), k=1,2, \ldots, m$,

(iii) $x(t) \in H$ has càdlàg paths on $t \in J$ a.e. and there exists a function $\sigma \in N_{F, x}$ such that

$$
\begin{aligned}
x(t)= & S(t)[\phi(0)-g(0, \phi, 0)]+g\left(t, x_{t}, \int_{0}^{t} a\left(t, s, x_{s}\right) d s\right) \\
& +\int_{0}^{t} A S(t-s) g\left(s, x_{s}, \int_{0}^{s} a\left(s, \tau, x_{\tau}\right) d \tau\right) d s+\int_{0}^{t} A S(t-s) f\left(s, x_{s}\right) d s \\
& +\int_{0}^{t} S(t-s) \sigma(s) d w(s)+\sum_{0<t_{k}<t} S\left(t-t_{k}\right) I_{k}\left(x\left(t_{k}^{-}\right)\right), \quad t \in J,
\end{aligned}
$$

(iv) $x_{0}(\cdot)=\phi \in L^{2}\left(\Omega, \mathcal{B}_{h}\right)$ on $J_{0}=(-\infty, 0]$ satisfies $\|\phi\|_{\mathcal{B}_{h}}^{2}<\infty$.

Now, we assume the following hypotheses:

(H1) $A$ is the infinitesimal generator of a compact analytic resolvent operator $S(t), t \geq 0$, in the Hilbert space $H$ and there exist positive constants $M$ and $M_{1}$ such that

$$
\|S(t)\|^{2} \leq M, \quad\left\|A^{-\beta}\right\| \leq M_{1}, \quad t \in J
$$

(H2) $a: D \times \mathcal{B}_{h} \rightarrow H, D=\{(t, s) \in J \times J: t \geq s\}$ is a continuous function and there exists a constant $M_{a}$ such that

$$
E\left|\int_{0}^{t}[a(t, s, x)-a(t, s, y)] d s\right|^{2} \leq M_{a}\|x-y\|_{\mathcal{B}_{h}}^{2} \quad \text { for all } t \in J, x, y \in \mathcal{B}_{h} .
$$

(H3) There exist constants $0<\beta<1$ and $M_{g}$ such that $g$ is $H_{\beta}$-valued, $(-A)^{\beta} g$ is continuous and

$$
E\left|(-A)^{\beta} g\left(t, x_{1}, y_{1}\right)-(-A)^{\beta} g\left(t, x_{2}, y_{2}\right)\right|^{2} \leq M_{g}\left[\left\|x_{1}-x_{2}\right\|_{\mathcal{B}_{h}}^{2}+E\left|y_{1}-y_{2}\right|^{2}\right] .
$$

(H4) The function $f: J \times \mathcal{B}_{h} \rightarrow H$ satisfies the following conditions:

(i) $t \mapsto f(t, s)$ is measurable for each $x \in \mathcal{B}_{h}$;

(ii) $x \mapsto f(t, x)$ is continuous for almost all $t \in J$;

(iii) There exists a constant $M_{f}$ such that

$$
E\left|(-A)^{\beta} f(t, x)-(-A)^{\beta} f(t, y)\right|^{2} \leq M_{f}\|x-y\|_{\mathcal{B}_{h}}^{2}
$$

for all $x, y \in \mathcal{B}_{h}, t \in J$ and

$$
E|f(t, x)|^{2} \leq p(t) \psi\left(\|x\|_{\mathcal{B}_{h}}^{2}\right)
$$

for almost all $t \in J$, where $p \in L^{1}(J, \mathbb{R}), \psi: \mathbb{R}_{+} \rightarrow(0, \infty)$ is continuous and increasing with

$$
\begin{aligned}
& \int_{0}^{b} \overline{\mu(s)} d s \leq \int_{B_{0} k_{1}}^{\infty} \frac{1}{\psi(s)} d s, \\
& \bar{\mu}(t)=B_{0} k_{3} p(t),
\end{aligned}
$$




$$
\begin{aligned}
& k_{1}=\frac{4\|\phi\|_{\mathcal{B}_{h}}^{2}+l^{2} F}{1-96 l^{2}\left\|(-A)^{-\beta}\right\|^{2} M_{g}\left(1+2 M_{a}\right)}, \\
& k_{2}=\frac{96 b l^{2} M_{g}\left(1+2 M_{a}\right) c_{1-\beta}^{2}}{1-96 l^{2}\left\|(-A)^{-\beta}\right\|^{2} M_{g}\left(1+2 M_{a}\right)}, \\
& k_{3}=\frac{48 M b l^{2}}{1-96 l^{2}\left\|(-A)^{-\beta}\right\|^{2} M_{g}\left(1+2 M_{a}\right)}, \\
& L_{0}=3 l^{2}\left[M_{g}\left(1+M_{a}\right)\left(\left\|(-A)^{-\beta}\right\|^{2}+\frac{\left(C_{1-\beta} b^{\beta}\right)^{2}}{2 \beta-1}\right)+M_{f} \frac{\left(C_{1-\beta} b^{\beta}\right)^{2}}{2 \beta-1}\right]<1, \\
& B_{0}=e^{k_{2}^{n} \Gamma(\beta)^{n} b^{n \beta} / \Gamma(n \beta)} \sum_{j=1}^{n-1}\left(\frac{k_{2} b^{\beta}}{\beta}\right)^{j}, \\
& c_{1}=b^{2} \sup _{(t, s) \in D} a^{2}(t, s, 0), \quad c_{2}=\left\|(-A)^{\beta}\right\|^{2} \sup _{t \in J}\|g(t, 0,0)\|^{2}
\end{aligned}
$$

and

$$
\begin{aligned}
\mathcal{F}= & 4 M|\phi(0)|^{2}+96\left(M+\left\|(-A)^{-\beta}\right\|^{2}\right) c_{2}+192\left\|(-A)^{-\beta}\right\|^{2} M_{g} c_{1} \\
& +\frac{192 b^{2 \beta} C_{1-\beta}^{2}}{2 \beta-1}\left(c_{2}+2 M_{g} c_{1}\right)+48 M\|\mu\|_{L_{\mathrm{loc}}^{1}\left(J, \mathbb{R}^{+}\right)} b^{2} \operatorname{Tr}(Q) \\
& +48 M m^{2} \sum_{k=1}^{m} d_{k}+96 M\left\|(-A)^{-\beta}\right\|^{2} M_{g}\|\phi\|_{\mathcal{B}_{h}}^{2} .
\end{aligned}
$$

(H5) The multi-valued mapping $F: J \times \mathcal{B}_{h} \rightarrow \mathcal{P}_{b d, c l, c v}(L(K, H))$ is an $L^{2}$-Carathéodory function that satisfies the following conditions:

(i) For each $t \in J$, the function $F(t, \cdot): \mathcal{B}_{h} \rightarrow \mathcal{P}_{b d, c l, c v}(L(K, H))$ is u.s.c. and for each fixed $x \in \mathcal{B}_{h}$, the function $F(\cdot, x)$ is measurable. For each $x \in \mathcal{B}_{h}$, the set

$$
N_{F, x}=\left\{\sigma \in L^{2}(K, H): \sigma(t) \in F(t, x) \text { for a.e. } t \in J\right\}
$$

is nonempty.

(ii) There exists a positive function $\mu \in L_{\text {loc }}^{1}\left(J, \mathbb{R}^{+}\right)$such that

$$
\|F(t, x)\|^{2}=\sup _{\sigma \in F(t, x)} E|\sigma|^{2} \leq \mu(t) .
$$

(H6) $I_{k} \in C\left(H_{\alpha}, H_{\alpha}\right)$ and there exist positive constants $d_{k}$ such that for each $x \in H_{\alpha}$,

$$
\left|I_{k}(x)\right|^{2} \leq d_{k}, \quad k=1,2, \ldots, m
$$

We consider the mapping $\Phi: \mathcal{B}_{h} \rightarrow \mathcal{P}\left(\mathcal{B}_{h}\right)$ defined by

$$
\Phi x(t)=\left\{\begin{array}{l}
\phi(t), \quad t \in(-\infty, 0], \\
S(t)[\phi(0)-g(0, \phi, 0)]+g\left(t, x_{t}, \int_{0}^{t} a\left(t, s, x_{s}\right) d s\right) \\
\quad+\int_{0}^{t} A S(t-s) g\left(s, x_{s}, \int_{0}^{s} a\left(s, \tau, x_{\tau}\right) d \tau\right) d s \\
\quad+\int_{0}^{t} A S(t-s) f\left(s, x_{s}\right) d s+\int_{0}^{t} S(t-s) \sigma(s) d w(s) \\
\quad+\sum_{0<t_{k}<t} S\left(t-t_{k}\right) I_{k}\left(x\left(t_{k}^{-}\right)\right), \quad t \in J,
\end{array}\right.
$$


where $\sigma \in N_{F, x}$. For each $\phi \in \mathcal{B}_{h}$, we define

$$
\tilde{\phi}(t)= \begin{cases}\phi(t), & t \in(-\infty, 0], \\ S(t) \phi(0), & t \in J,\end{cases}
$$

and then $\tilde{\phi} \in \mathcal{B}_{h}$. Let $x(t)=y(t)+\tilde{\phi}(t), t \in(-\infty, b]$. Then it is easy to see that $x$ satisfies (1.1)-(1.3) if and only if $y$ satisfies $y_{0}=0$ and

$$
\begin{aligned}
y(t)= & -S(t) g(0, \phi, 0)+g\left(t, y_{t}+\tilde{\phi}_{t}, \int_{0}^{t} a\left(t, s, y_{s}+\tilde{\phi}_{s}\right) d s\right) \\
& +\int_{0}^{t} A S(t-s) g\left(s, y_{s}+\tilde{\phi}_{s}, \int_{0}^{s} a\left(s, \tau, y_{\tau}+\tilde{\phi}_{\tau}\right) d \tau\right) d s \\
& +\int_{0}^{t} A S(t-s) f\left(s, y_{s}+\phi_{s}\right) d s+\int_{0}^{t} S(t-s) \sigma(s) d w(s) \\
& +\sum_{0<t_{k}<t} S\left(t-t_{k}\right) I_{k}\left(y\left(t_{k}^{-}\right)+\tilde{\phi}\left(t_{k}^{-}\right)\right), \quad t \in J,
\end{aligned}
$$

where $\sigma \in N_{F, y}$. Let $\mathcal{B}_{h}^{\prime}=\left\{y \in \mathcal{B}_{h}: y_{0}=0 \in \mathcal{B}_{h}\right\}$. For any $y \in \mathcal{B}_{h}^{\prime}$,

$$
\begin{aligned}
\|y\|_{b} & =\left\|y_{0}\right\|_{\mathcal{B}_{h}}+\sup _{0 \leq s \leq b}\left(E|y(s)|^{2}\right)^{\frac{1}{2}} \\
& =\sup _{0 \leq s \leq b}\left(E|y(s)|^{2}\right)^{\frac{1}{2}}
\end{aligned}
$$

and thus $\left(\mathcal{B}_{h}^{\prime},\|\cdot\|_{b}\right)$ is a Banach space. Set $\mathcal{B}_{q}=\left\{y \in \mathcal{B}_{h}^{\prime}:\|y\|_{b}^{2} \leq q\right\}$ for some $q \geq 0$. Then $\mathcal{B}_{q} \subseteq \mathcal{B}_{h}^{\prime}$ is uniformly bounded and for any $y \in \mathcal{B}_{q}$, from Lemma 2.2 , we see that

$$
\begin{aligned}
\left\|y_{t}+\tilde{\phi}_{t}\right\|_{\mathcal{B}_{h}}^{2} \leq & 2\left\|y_{t}\right\|_{\mathcal{B}_{h}}^{2}+2\left\|\tilde{\phi}_{t}\right\|_{\mathcal{B}_{h}}^{2} \\
\leq & 4 l^{2} \sup _{0 \leq s \leq t} E|y(s)|^{2}+4\left\|y_{0}\right\|_{\mathcal{B}_{h}}^{2} \\
& +4 l^{2} \sup _{0 \leq s \leq t}\|\tilde{\phi}(s)\|^{2}+4\left\|\tilde{\phi}_{0}\right\|_{\mathcal{B}_{h}}^{2} \\
\leq & 4 l^{2}\left(q+M|\phi(0)|^{2}\right)+4\|\tilde{\phi}\|_{\mathcal{B}_{h}}^{2} \\
:= & q^{\prime} .
\end{aligned}
$$

Define the operator $\tilde{\Phi}: \mathcal{B}_{h}^{\prime} \rightarrow \mathcal{P}\left(\mathcal{B}_{h}^{\prime}\right)$ by

$$
\tilde{\Phi} y(t)=\left\{\begin{aligned}
0, \quad t \in(-\infty, 0], \\
-S(t) g(0, \phi, 0)+g\left(t, y_{t}+\tilde{\phi}_{t}, \int_{0}^{t} a\left(t, s, y_{s}+\tilde{\phi}_{s}\right) d s\right) \\
\quad+\int_{0}^{t} A S(t-s) g\left(s, y_{s}+\tilde{\phi}, \int_{0}^{s} a\left(s, \tau, y_{\tau}+\tilde{\phi}_{\tau}\right) d \tau\right) d s \\
\quad+\int_{0}^{t} A S(t-s) f\left(s, y_{s}+\tilde{\phi}_{s}\right) d s+\int_{0}^{t} S(t-s) \sigma(s) d w(s) \\
\quad+\sum_{0<t_{k}<t} S\left(t-t_{k}\right) I_{k}\left(y\left(t_{k}^{-}\right)+\tilde{\phi}\left(t_{k}^{-}\right)\right), \quad t \in J,
\end{aligned}\right.
$$


where $\sigma \in N_{F, y}$. Obviously, the operator $\Phi$ has a fixed point is equivalent to proving that $\tilde{\Phi}$ has a fixed point. Now, we decompose $\tilde{\Phi}$ as $\tilde{\Phi}_{1}+\tilde{\Phi}_{2}$, where

$$
\begin{aligned}
\tilde{\Phi}_{1} y(t)= & -S(t) g(0, \phi, 0)+g\left(t, y_{t}+\tilde{\phi}_{t}, \int_{0}^{t} a\left(t, s, y_{s}+\tilde{\phi}_{s}\right) d s\right) \\
& +\int_{0}^{t} A S(t-s) g\left(s, y_{s}+\tilde{\phi}_{s}, \int_{0}^{s} a\left(s, \tau, y_{\tau}+\tilde{\phi}_{\tau}\right) d \tau\right) d s \\
& +\int_{0}^{t} A S(t-s) f\left(s, y_{s}+\tilde{\phi}_{s}\right) d s
\end{aligned}
$$

and

$$
\tilde{\Phi}_{2} y(t)=\int_{0}^{s} S(t-s) \sigma(s) d w(s)+\sum_{0<t_{k}<t} S\left(t-t_{k}\right) I_{k}\left(y\left(t_{k}^{-}\right)+\tilde{\phi}\left(t_{k}^{-}\right)\right), \quad t \in J
$$

where $\sigma \in N_{F, y}$. In what follows, we show that the operators $\tilde{\Phi}_{1}$ and $\tilde{\Phi}_{2}$ satisfy all the conditions of Theorem 2.1.

Lemma 3.1 Assume that the assumptions (H1)-(H6) hold. Then $\tilde{\Phi}_{1}$ is a contraction and $\tilde{\Phi}_{2}$ is u.s.c. and completely continuous.

Proof We give the proof in several steps:

Step 1. $\tilde{\Phi}_{1}$ is a contraction.

Let $u, v \in \mathcal{B}_{h}^{\prime}$. Then we have

$$
\begin{aligned}
E\left|\tilde{\phi}_{1} u(t)-\tilde{\phi}_{1} v(t)\right|^{2} \\
\leq 3 E\left|g\left(t, u_{t}+\tilde{\phi}_{t}, \int_{0}^{t} a\left(t, s, u_{s}+\tilde{\phi}_{s}\right) d s\right)-g\left(t, v_{t}+\tilde{\phi}_{t}, \int_{0}^{t} a\left(t, s, v_{s}+\tilde{\phi}_{s}\right) d s\right)\right|^{2} \\
+3 b E\left(\int_{0}^{t} \mid A S(t-s)\left[g\left(s, u_{s}+\tilde{\phi}_{s}, \int_{0}^{s} a\left(s, \tau, u_{\tau}+\tilde{\phi}_{\tau}\right) d \tau\right)\right.\right. \\
\left.\left.\quad-g\left(s, v_{s}+\tilde{\phi}_{s}, \int_{0}^{s} a\left(s, \tau, u_{\tau}+\tilde{\phi}_{\tau}\right) d \tau\right)\right]\left.\right|^{2} d s\right) \\
+3 b E\left(\int_{0}^{t}\left|A S(t-s)\left[f\left(s, u_{s}+\tilde{\phi}_{s}\right)-f\left(s, v_{s}+\tilde{\phi}_{s}\right)\right]\right|^{2} d s\right) \\
\leq 3\left\|(-A)^{-\beta}\right\|^{2} M_{g}\left(\left\|u_{t}-v_{t}\right\|_{\mathcal{B}_{h}}^{2}+M_{a}\left\|u_{t}-v_{t}\right\|_{\mathcal{B}_{h}}^{2}\right) \\
+3 b \int_{0}^{t} \frac{C_{1-\beta}^{2}}{(t-s)^{2(1-\beta)}} M_{g}\left(\left\|u_{s}-v_{s}\right\|_{\mathcal{B}_{h}}^{2}+M_{a}\left\|u_{s}-v_{s}\right\|_{\mathcal{B}_{h}}^{2}\right) d s \\
+3 b \int_{0}^{t} \frac{C_{1-\beta}^{2}}{(t-s)^{2(1-\beta)}} M_{f}\left\|u_{s}-v_{s}\right\|_{\mathcal{B}_{h}}^{2} d s \\
\leq 3\left\|(-A)^{-\beta}\right\|^{2} M_{g}\left(1+M_{a}\right)\left\|u_{t}-v_{t}\right\|_{\mathcal{B}_{h}}^{2} \\
+3 M_{g}\left(1+M_{a}\right) \frac{\left(C_{1-\beta} b^{\beta}\right)^{2}}{2 \beta-1}\left\|u_{t}-v_{t}\right\|_{\mathcal{B}_{h}}^{2} \\
+3 M_{f} \frac{\left(C_{1-\beta} b^{\beta}\right)^{2}}{2 \beta-1}\left\|u_{t}-v_{t}\right\|_{\mathcal{B}_{h}}^{2}
\end{aligned}
$$




$$
\begin{aligned}
\leq & 3\left[M_{g}\left(1+M_{a}\right)\left(\left\|(-A)^{-\beta}\right\|^{2}+\frac{\left(C_{1-\beta} b^{\beta}\right)^{2}}{2 \beta-1}\right)+M_{f} \frac{\left(C_{1-\beta} b^{\beta}\right)^{2}}{2 \beta-1}\right] \\
& \times\left[l^{2} \sup _{s \in[0, b]} E|u(s)-v(s)|^{2}+\left\|u_{0}\right\|_{\mathcal{B}_{h}}^{2}+\left\|v_{0}\right\|_{\mathcal{B}_{h}}^{2}\right] \\
= & 3 l^{2}\left[M_{g}\left(1+M_{a}\right)\left(\left\|(-A)^{-\beta}\right\|^{2}+\frac{\left(C_{1-\beta} b^{\beta}\right)^{2}}{2 \beta-1}\right)+M_{f} \frac{\left(C_{1-\beta} b^{\beta}\right)^{2}}{2 \beta-1}\right] \sup _{s \in[0, b]} E|u(s)-v(s)|^{2} \\
= & L_{0} \sup _{s \in[0, b]} E|u(s)-v(s)|^{2},
\end{aligned}
$$

where $L_{0}=3 l^{2}\left[M_{g}\left(1+M_{a}\right)\left(\left\|(-A)^{-\beta}\right\|^{2}+\frac{\left(C_{1-\beta} b^{\beta}\right)^{2}}{2 \beta-1}\right)+M_{f} \frac{\left(C_{1-\beta} b^{\beta}\right)^{2}}{2 \beta-1}\right]<1$ and we have used the fact that $\left\|u_{0}\right\|_{\mathcal{B}_{h}}^{2}=0$ and $\left\|v_{0}\right\|_{\mathcal{B}_{h}}^{2}=0$. Taking the supremum over $t$, we obtain

$$
\left\|\tilde{\phi}_{1} u-\tilde{\phi}_{1} v\right\|_{b}^{2} \leq L_{0}\|u-v\|_{b}^{2}
$$

and so $\tilde{\phi}_{1}$ is a contraction.

Now, we show that the operator $\tilde{\Phi}_{2}$ is completely continuous.

Step 2. $\tilde{\Phi}_{2} y$ is convex for each $y \in \mathcal{B}_{h}^{\prime}$.

In fact, if $u_{1}, u_{2} \in \tilde{\Phi}_{2}(y)$, then there exist $\sigma_{1}, \sigma_{2} \in N_{F, y}$ such that

$$
u_{i}(t)=\int_{0}^{t} S(t-s) \sigma_{i}(s) d w(s)+\sum_{0<t_{k}<t} S\left(t-t_{k}\right) I_{k}\left(y\left(t_{k}^{-}\right)+\tilde{\phi}\left(t_{k}^{-}\right)\right)
$$

for $i=1,2$ and $t \in J$. Let $\lambda \in[0,1]$. Then for each $t \in J$, we have

$$
\begin{aligned}
\lambda u_{1}(t)+(1-\lambda) u_{2}(t)= & \int_{0}^{t} S(t-s)\left[\lambda \sigma_{1}(s)+(1-\lambda) \sigma_{2}(s)\right] d w(s) \\
& +\sum_{0<t_{k}<t} S\left(t-t_{k}\right) I_{k}\left(y\left(t_{k}^{-}\right)+\tilde{\phi}\left(t_{k}^{-}\right)\right) .
\end{aligned}
$$

Since $N_{F, y}$ is convex (because $F$ has convex values), we obtain

$$
\lambda u_{1}(t)+(1-\lambda) u_{2}(t) \in \tilde{\Phi}_{2}(y) .
$$

Step 3. $\tilde{\Phi}_{2}$ maps bounded sets into bounded sets in $\mathcal{B}_{h}^{\prime}$.

It is enough to show that there exists a positive constant $\Lambda$ such that for each $u \in \tilde{\Phi}_{2} y$, $y \in \mathcal{B}_{q}=\left\{y \in \mathcal{B}_{h}^{\prime}:\|y\|_{b} \leq q\right\}$ one has $\|u\|_{b} \leq \Lambda$. If $u \in \tilde{\Phi}_{2}(y)$, there exists $\sigma \in N_{F, y}$ such that for each $t \in J$

$$
u(t)=\int_{0}^{t} S(s-t) \sigma(s) d w(s)+\sum_{0<t_{k}<t} S\left(t-t_{k}\right) I_{k}\left(y\left(t_{k}^{-}\right)+\tilde{\phi}\left(t_{k}^{-}\right)\right)
$$

and so

$$
\begin{aligned}
E|u(t)|^{2} & =E\left|\int_{0}^{t} S(t-s) \sigma(s) d w(s)+\sum_{0<t_{k}<t} S\left(t-t_{k}\right) I_{k}\left(y\left(t_{k}^{-}\right)+\tilde{\phi}\left(t_{k}^{-}\right)\right)\right|^{2} \\
& \leq 2 E\left|\int_{0}^{t} S(t-s) \sigma(s) d w(s)\right|^{2}+2 E\left|\sum_{0<t_{k}<t} S\left(t-t_{k}\right) I_{k}\left(y\left(t_{k}^{-}\right)+\tilde{\phi}\left(t_{k}^{-}\right)\right)\right|^{2}
\end{aligned}
$$




$$
\begin{aligned}
& \leq 2 \operatorname{Tr}(Q) M b \int_{0}^{b} \mu(s) d s+2 M m^{2} \sum_{k=1}^{m} d_{k} \\
& \leq 2 \operatorname{Tr}(Q) M b^{2}\|\mu\|_{L_{\mathrm{loc}}^{1}\left(J, \mathbb{R}^{+}\right)}+2 M m^{2} \sum_{k=1}^{m} d_{k} \\
& :=\Lambda .
\end{aligned}
$$

Thus, for each $y \in \mathcal{B}_{h}^{\prime}$, we get $\|u\|_{b}^{2} \leq \Lambda$.

Step 4. $\tilde{\Phi}_{2}$ maps bounded sets into equicontinuous sets of $\mathcal{B}_{h}^{\prime}$.

Let $0<\tau_{1}<\tau_{2} \leq b$. For each $y \in \mathcal{B}_{q}=\left\{y \in \mathcal{B}_{h}^{\prime}:\|y\|_{b} \leq q\right\}$ and $u \in \tilde{\Phi}_{2}(y)$. Let $\tau_{1}, \tau_{2} \in$ $J \backslash\left\{t_{1}, t_{2}, \ldots, t_{m}\right\}$. Then there exists $\sigma \in N_{F, y}$ such that for each $t \in J$,

$$
u(t)=\int_{0}^{t} S(t-s) \sigma(s) d w(s)+\sum_{0<t_{k}<t} S\left(t-t_{k}\right) I_{k}\left(y\left(t_{k}^{-}\right)+\tilde{\phi}\left(t_{k}^{-}\right)\right) .
$$

Thus we have

$$
\begin{aligned}
E\left|u\left(\tau_{2}\right)-u\left(\tau_{1}\right)\right|^{2} & =E \mid \int_{0}^{\tau_{2}} S\left(\tau_{2}-s\right) \sigma(s) d w(s)+\sum_{0<t_{k}<\tau_{2}} S\left(\tau_{2}-s\right) I_{k}\left(y\left(t_{k}^{-}\right)+\tilde{\phi}\left(t_{k}^{-}\right)\right) \\
& -\int_{0}^{\tau_{1}} S\left(\tau_{1}-s\right) \sigma(s) d w(s)-\left.\sum_{0<t_{k}<\tau_{1}} S\left(\tau_{1}-t_{k}\right) I_{k}\left(y\left(t_{k}^{-}\right)+\tilde{\phi}\left(t_{k}^{-}\right)\right)\right|^{2} \\
\leq & 2 E \mid \int_{0}^{\tau_{1}-\varepsilon}\left(S\left(\tau_{2}-s\right) \sigma(s)-S\left(\tau_{1}-s\right) \sigma(s)\right) d w(s) \\
& +\int_{\tau_{1}-\varepsilon}^{\tau_{1}}\left(S\left(\tau_{2}-s\right) \sigma(s)-S\left(\tau_{1}-s\right) \sigma(s)\right) d w(s)+\left.\int_{\tau_{1}}^{\tau_{2}} S\left(\tau_{2}-s\right) \sigma(s) d w(s)\right|^{2} \\
& +2 E \mid \sum_{0<t_{k}<\tau_{1}}\left[S\left(\tau_{2}-t_{k}\right)-S\left(\tau_{1}-t_{k}\right)\right] I_{k}\left(y\left(t_{k}^{-}\right)+\tilde{\phi}\left(t_{k}^{-}\right)\right) \\
& +\left.\sum_{\tau_{1}<t_{k}<\tau_{2}} S\left(\tau_{2}-t_{k}\right) I_{k}\left(y\left(t_{k}^{-}\right)+\tilde{\phi}\left(t_{k}^{-}\right)\right)\right|^{2} \\
\leq & 6 \varepsilon \operatorname{Tr}(Q) \int_{0}^{\tau_{1}-\varepsilon} \mu(s)\left\|S\left(\tau_{2}-s\right)-S\left(\tau_{1}-s\right)\right\|^{2} d s \\
& +6 \varepsilon \operatorname{Tr}(Q) \int_{\tau_{1}-\varepsilon}^{\tau_{1}} \mu(s)\left\|S\left(\tau_{2}-s\right)-S\left(\tau_{1}-s\right)\right\|^{2} d s \\
& +6\left(\tau_{2}-\tau_{1}\right) \operatorname{Tr}(Q) \int_{\tau_{1}}^{\tau_{2}} \mu(s)\left\|S\left(\tau_{2}-s\right)\right\|^{2} d s \\
& +4 m^{2} \sum_{0<t_{k}<\tau_{1}}\left\|S\left(\tau_{2}-s\right)-S\left(\tau_{1}-s\right)\right\|^{2} d_{k} \\
& +4 m^{2} M \sum_{\tau_{1}<t_{k}<\tau_{2}} d_{k} . \\
&
\end{aligned}
$$

The right-hand side of the above inequality tends to zero as $\tau_{1} \rightarrow \tau_{2}$ with $\varepsilon$ sufficiently small, since $S(t)$ is strongly continuous and the compactness of $S(t)$ for $t>0$ implies the 
continuity in the uniform operator topology. Thus, the set $\left\{\tilde{\Phi}_{2} y: y \in \mathcal{B}_{q}\right\}$ is equicontinuous. Here we consider the case $0<\tau_{1}<\tau_{2} \leq b$, since the case $\tau_{1}<\tau_{2} \leq 0$ or $\tau_{1} \leq 0 \leq \tau_{2} \leq b$ is simple.

Step 5. $\tilde{\Phi}_{2}$ maps $\mathcal{B}_{q}$ into a precompact set in $H$.

Let $0<t \leq b$ and $0<\varepsilon<t$. For $y \in \mathcal{B}_{q}$ and $u \in \tilde{\Phi}_{2}(y)$, there exists $\sigma \in N_{F, y}$ such that

$$
\begin{aligned}
u(t)= & \int_{0}^{t-\varepsilon} S(t-s) \sigma(s) d w(s)+\int_{t-\varepsilon}^{t} S(t-s) \sigma(s) d w(s) \\
& +\sum_{0<t_{k}<t} S\left(t-t_{k}\right) I_{k}\left(y\left(t_{k}^{-}\right)+\tilde{\phi}\left(t_{k}^{-}\right)\right) .
\end{aligned}
$$

Define

$$
u_{\varepsilon}(t)=S(\varepsilon) \int_{0}^{t-\varepsilon} S(t-\varepsilon-s) \sigma(s) d w(s)+\sum_{0<t_{k}<t-\varepsilon} S\left(t-t_{k}\right) I_{k}\left(y\left(t_{k}^{-}\right)+\tilde{\phi}\left(t_{k}^{-}\right)\right) .
$$

Since $S(t)$ is a compact operator, the set $V_{\varepsilon}(t)=\left\{u_{\varepsilon}(t): u_{\varepsilon} \in \tilde{\Phi}_{2}\left(\mathcal{B}_{q}\right)\right\}$ is relatively compact in $H$ for each $\varepsilon, 0<\varepsilon<t$. Moreover,

$$
\begin{aligned}
& E\left|u(t)-u_{\varepsilon}(t)\right|^{2} \\
& =E \mid \int_{0}^{t-\varepsilon} S(t-s) \sigma(s) d w(s)+\int_{t-\varepsilon}^{t} S(t-s) \sigma(s) d w(s) \\
& \quad+\sum_{0<t_{k}<t} S\left(t-t_{k}\right) I_{k}\left(y\left(t_{k}^{-}\right)+\tilde{\phi}\left(t_{k}^{-}\right)\right)-S(\varepsilon) \int_{0}^{t-\varepsilon} S(t-\varepsilon-s) \sigma(s) d w(s) \\
& \quad-\left.\sum_{0<t_{k}<t-\varepsilon} S\left(t-t_{k}\right) I_{k}\left(y\left(t_{k}^{-}\right)+\tilde{\phi}\left(t_{k}^{-}\right)\right)\right|^{2} \\
& \leq 4 M b \operatorname{Tr}(Q) \varepsilon\|\mu\|_{L_{10 c}^{1}\left(J, \mathbb{R}^{+}\right)}+4 m^{2} M \sum_{t-\varepsilon<t_{k}<t} d_{k} .
\end{aligned}
$$

Therefore letting $\varepsilon \rightarrow 0$, we can see that there are relative compact sets arbitrarily close to the set $\left\{u(t): u \in \tilde{\Phi}_{2}\left(B_{q}\right)\right\}$. Thus, the set $\left\{u(t): u \in \tilde{\Phi}_{2}\left(B_{q}\right)\right\}$ is relatively compact in $H$. Hence, the Arzelá-Ascoli theorem shows that $\tilde{\Phi}_{2}$ is a compact multi-valued mapping.

Step 6. $\tilde{\Phi}_{2}$ has a closed graph.

Let $y_{n} \rightarrow y_{*}, u_{n} \in \tilde{\Phi}_{2}\left(y_{n}\right)$ and $u_{n} \rightarrow u_{*}$. We prove that $u_{*} \in \tilde{\Phi}_{2}\left(y_{*}\right)$.

Indeed, $u_{n} \in \tilde{\Phi}_{2}\left(y_{n}\right)$ means that there exists $\sigma_{n} \in N_{F, y_{n}}$ such that

$$
u_{n}(t)=\int_{0}^{t} S(t-s) \sigma_{n}(s) d w(s)+\sum_{0<t_{k}<t} S\left(t-t_{k}\right) I_{k}\left(y_{n}\left(t_{k}^{-}\right)+\tilde{\phi}\left(t_{k}^{-}\right)\right), \quad t \in J .
$$

Thus we must prove that there exists $\sigma_{*} \in N_{F, y_{*}}$ such that

$$
u_{*}(t)=\int_{0}^{t} S(t-s) \sigma_{*}(s) d w(s)+\sum_{0<t_{k}<t} S\left(t-t_{k}\right) I_{k}\left(y_{*}\left(t_{k}^{-}\right)+\tilde{\phi}\left(t_{k}^{-}\right)\right), \quad t \in J .
$$


Since $I_{k}, k=1,2, \ldots, m$, are continuous, we see that

$$
\left\|\sum_{0<t_{k}<t} S\left(t-t_{k}\right) I_{k}\left(y_{n}\left(t_{k}^{-}\right)+\tilde{\phi}\left(t_{k}^{-}\right)\right)-\sum_{0<t_{k}<t} S\left(t-t_{k}\right) I_{k}\left(y_{*}\left(t_{k}^{-}\right)+\tilde{\phi}\left(t_{k}^{-}\right)\right)\right\|_{b}^{2} \rightarrow 0
$$

as $n \rightarrow \infty$. Consider the linear continuous operator $\Gamma: L^{2}(J, H) \rightarrow C(J, H)$ with $\Gamma(\sigma)(t)=$ $\int_{0}^{t} S(t-s) \sigma(s) d w(s)$, where $\sigma \in N_{F, y}$. From Lemma 2.3, it follows that $\Gamma \circ N_{F}$ is a closed graph operator. Moreover, we have

$$
u_{n}(t)-\sum_{0<t_{k}<t} S\left(t-t_{k}\right) I_{k}\left(y_{n}\left(t_{k}^{-}\right)+\tilde{\phi}\left(t_{k}^{-}\right)\right) \in \Gamma\left(N_{F, y_{n}}\right)
$$

Since $y_{n} \rightarrow y_{*}$, from Lemma 2.3, we obtain

$$
u_{*}(t)-\sum_{0<t_{k}<t} S\left(t-t_{k}\right) I_{k}\left(y_{*}\left(t_{k}^{-}\right)+\tilde{\phi}\left(t_{k}^{-}\right)\right) \in \Gamma\left(N_{F, y_{*}}\right)
$$

That is, there exists a $\sigma_{*} \in N_{F, y_{*}}$ such that

$$
\begin{aligned}
u_{*}(t)-\sum_{0<t_{k}<t} S\left(t-t_{k}\right) I_{k}\left(y_{*}\left(t_{k}^{-}\right)+\tilde{\phi}\left(t_{k}^{-}\right)\right) & =\Gamma\left(\sigma_{*}(t)\right) \\
& =\int_{0}^{t} S(t-s) \sigma_{*}(s) d w(s) .
\end{aligned}
$$

Therefore $\tilde{\Phi}_{2}$ has a closed graph and $\tilde{\Phi}_{2}$ is u.s.c. This completes the proof.

Lemma 3.2 Assume that the assumptions (H1)-(H2) hold. Then there exists a constant $K>0$ such that $\left\|y_{t}+\tilde{\phi}_{t}\right\|_{\mathcal{B}_{h}}^{2} \leq K$ for all $t \in J$, where $K$ is depends only on $b$ and the functions $\psi$ and $\bar{\mu}$.

Proof Let $y$ be a possible solution of $y \in \lambda \tilde{\Phi}(y)$ for some $0<\lambda<1$. Then there exists $\sigma \in$ $N_{F, y}$ such that for $t \in J$ we have

$$
\begin{aligned}
y(t)= & -\lambda S(t) g(0, \phi, 0)+\lambda g\left(t, y_{t}+\tilde{\phi}_{t}, \int_{0}^{t} a\left(t, s, y_{s}+\tilde{\phi}_{s}\right) d s\right) \\
& +\lambda \int_{0}^{t} A S(t-s) g\left(s, y_{s}+\tilde{\phi}_{s}, \int_{0}^{s} a\left(s, \tau, y_{\tau}+\tilde{\phi}_{\tau}\right) d \tau\right) d s \\
& +\lambda \int_{0}^{t} S(t-s) f\left(s, y_{s}+\tilde{\phi}_{s}\right) d s+\int_{0}^{t} S(t-s) \sigma(s) d w(s) \\
& +\lambda \sum_{0<t_{k}<t} S\left(t-t_{k}\right) I_{k}\left(y\left(t_{k}^{-}\right)+\tilde{\phi}\left(t_{k}^{-}\right)\right) .
\end{aligned}
$$

Then, by the assumptions, we deduce that

$$
\begin{aligned}
E|y(t)|^{2} \leq & E \mid-S(t) g(0, \phi, 0)+g\left(t, y_{t}+\tilde{\phi}_{t}, \int_{0}^{t} a\left(t, s, y_{s}+\tilde{\phi}_{s}\right) d s\right) \\
& +\int_{0}^{t} A S(t-s) g\left(s, y_{s}+\tilde{\phi}_{s}, \int_{0}^{s} a\left(s, \tau, y_{\tau}+\tilde{\phi}_{\tau}\right) d \tau\right) d s
\end{aligned}
$$




$$
\begin{aligned}
& +\int_{0}^{t} S(t-s) f\left(s, y_{s}+\tilde{\phi}_{s}\right) d s+\int_{0}^{t} S(t-s) \sigma(s) d w(s) \\
& +\left.\sum_{0<t_{k}<t} S\left(t-t_{k}\right) I_{k}\left(y\left(t_{k}^{-}\right)+\tilde{\phi}\left(t_{k}^{-}\right)\right)\right|^{2} \\
\leq & 12\left\{2 M\left(\left\|(-A)^{-\beta}\right\|^{2} M_{g}\|\phi\|_{B_{h}}^{2}+c_{2}\right)\right. \\
& +2\left\|(-A)^{-\beta}\right\|^{2}\left[M_{g}\left(\left\|y_{s}+\tilde{\phi}_{s}\right\|_{\mathcal{B}_{h}}^{2}+2 M_{a}\left\|y_{s}+\tilde{\phi}_{s}\right\|_{\mathcal{B}_{h}}^{2}+2 c_{1}\right)+c_{2}\right] \\
& +2 b \int_{0}^{t} \frac{c_{1-\beta}^{2}}{(t-s)^{2(1-\beta)}}\left[M_{g}\left(\left\|y_{s}+\tilde{\phi}_{s}\right\|_{\mathcal{B}_{h}}^{2}+2 M_{a}\left\|y_{s}+\tilde{\phi}_{s}\right\|_{\mathcal{B}_{h}}^{2}+2 c_{1}\right)+c_{2}\right] d s \\
& \left.+M b \int_{0}^{t} p(s) \psi\left(\left\|y_{s}+\tilde{\phi}_{s}\right\|_{\mathcal{B}_{h}}^{2}\right) d s+M\|\mu\|_{L_{\mathrm{loc}}^{1}\left(J, \mathbb{R}^{+}\right)} b^{2} \operatorname{Tr}(Q)+M m^{2} \sum_{k=1}^{m} d_{k}\right\} \\
= & 24\left(M+\left\|(-A)^{-\beta}\right\|^{2}\right) c_{2}+48\left\|(-A)^{-\beta}\right\|^{2} M_{g} c_{1}+\frac{48 b^{2 \beta} c_{1-\beta}^{2}}{2 \beta-1}\left(c_{2}+2 M_{g} c_{1}\right) \\
& +12 M\|\mu\|_{L_{\mathrm{loc}}^{1}\left(J, \mathbb{R}^{+}\right)} b^{2} \operatorname{Tr}(Q)+12 M m^{2} \sum_{k=1}^{m} d_{k}+24 M\left\|(-A)^{-\beta}\right\|^{2} M_{g}\|\phi\|_{\mathcal{B}_{h}}^{2} \\
& +24\left\|(-A)^{-\beta}\right\|^{2} M_{g}\left(1+2 M_{a}\right)\left\|y_{s}+\tilde{\phi}_{s}\right\|_{\mathcal{B}_{h}}^{2} \\
& +24 b M_{g}\left(1+2 M_{a}\right) c_{1-\beta}^{2} \int_{0}^{t} \frac{\left\|y_{s}+\tilde{\phi}_{s}\right\|_{\mathcal{B}_{h}}^{2}}{(t-s)^{2(1-\beta)}} d s \\
& +12 M b \int_{0}^{t} p(s) \psi\left(\left\|y_{s}+\tilde{\phi}_{s}\right\|_{\mathcal{B}_{h}}^{2}\right) d s . \\
&
\end{aligned}
$$

From Lemma 2.2 we see that

$$
\left\|y_{t}+\tilde{\phi}_{t}\right\|_{\mathcal{B}_{h}}^{2} \leq 4 l^{2} \sup _{0 \leq s \leq t} E|y(s)|^{2}+4 l^{2} M|\tilde{\phi}(0)|^{2}+4\|\tilde{\phi}\|_{\mathcal{B}_{h}}^{2} .
$$

Thus, for any $t \in J$, we have

$$
\begin{aligned}
\| y_{t}+ & \tilde{\phi}_{t} \|_{\mathcal{B}_{h}}^{2} \\
\leq & 4 l^{2} M|\tilde{\phi}(0)|^{2}+4\|\tilde{\phi}\|_{\mathcal{B}_{h}}^{2}+96 l^{2}\left(M+\left\|(-A)^{-\beta}\right\|^{2}\right) c_{2} \\
& +192 l^{2}\left\|(-A)^{-\beta}\right\|^{2} M_{g} c_{1}+\frac{192 l^{2} b^{2 \beta} C_{1-\beta}^{2}}{2 \beta-1}\left(c_{2}+2 M_{g} c_{1}\right) \\
& +48 M\|\mu\|_{L_{\mathrm{loc}}^{1}\left(J, \mathbb{R}^{+}\right)} b^{2} l^{2} \operatorname{Tr}(Q)+48 M l^{2} m^{2} \sum_{k=1}^{m} d_{k} \\
& +96 M l^{2}\left\|(-A)^{-\beta}\right\|^{2} M_{g}\|\phi\|_{\mathcal{B}_{h}}^{2} \\
& +96 l^{2}\left\|(-A)^{-\beta}\right\|^{2} M_{g}\left(1+2 M_{a}\right)\left\|y_{s}+\tilde{\phi}_{s}\right\|_{\mathcal{B}_{h}}^{2} \\
& +96 b l^{2} M_{g}\left(1+2 M_{a}\right) C_{1-\beta}^{2} \int_{0}^{t} \frac{\left\|y_{s}+\tilde{\phi}_{s}\right\|_{\mathcal{B}_{h}}^{2}}{(t-s)^{2(1-\beta)}} d s \\
& +48 M b l^{2} \int_{0}^{t} p(s) \psi\left(\left\|y_{s}+\tilde{\phi}_{s}\right\|_{\mathcal{B}_{h}}^{2}\right) d s
\end{aligned}
$$




$$
\begin{aligned}
= & 4\|\phi\|_{\mathcal{B}_{h}}^{2}+l^{2} \mathcal{F}+96 l^{2}\left\|(-A)^{-\beta}\right\|^{2} M_{g}\left(1+2 M_{a}\right) \sup _{0 \leq s \leq t}\left\|y_{s}+\tilde{\phi}_{s}\right\|_{\mathcal{B}_{h}}^{2} \\
& +96 b l^{2} M_{g}\left(1+2 M_{a}\right) C_{1-\beta}^{2} \int_{0}^{t} \frac{\left\|y_{s}+\tilde{\phi}_{s}\right\|_{\mathcal{B}_{h}}^{2}}{(t-s)^{2(1-\beta)}} d s \\
& +48 M b l^{2} \int_{0}^{t} p(s) \psi\left(\left\|y_{s}+\tilde{\phi}_{s}\right\|_{\mathcal{B}_{h}}^{2}\right) d s .
\end{aligned}
$$

Let $v(t)=\sup _{0 \leq s \leq t}\left\|y_{s}+\tilde{\phi}_{s}\right\|_{\mathcal{B}_{h}}^{2}$. Then the function $v(t)$ is nondecreasing in $J$. Thus, we obtain

$$
\begin{aligned}
v(t) \leq & 4\|\phi\|_{\mathcal{B}_{h}}^{2}+l^{2} \mathcal{F}+96 l^{2}\left\|(-A)^{-\beta}\right\|^{2} M_{g}\left(1+2 M_{a}\right) v(t) \\
& +96 b l^{2} M_{g}\left(1+2 M_{a}\right) C_{1-\beta}^{2} \int_{0}^{t} \frac{v(s)}{(t-s)^{2(1-\beta)}} d s \\
& +48 M b l^{2} \int_{0}^{t} p(s) \psi(v(s)) d s .
\end{aligned}
$$

From this we derive that

$$
\begin{aligned}
v(t) \leq & \frac{4\|\phi\|_{\mathcal{B}_{h}}^{2}+l^{2} \mathcal{F}}{1-96 l^{2}\left\|(-A)^{-\beta}\right\|^{2} M_{g}\left(1+2 M_{a}\right)} \\
& +\frac{96 b l^{2} M_{g}\left(1+2 M_{a}\right) C_{1-\beta}^{2}}{1-96 l^{2}\left\|(-A)^{-\beta}\right\|^{2} M_{g}\left(1+2 M_{a}\right)} \int_{0}^{t} \frac{v(s)}{(t-s)^{1-\beta}} d s \\
& +\frac{48 M b l^{2}}{1-96 l^{2}\left\|(-A)^{-\beta}\right\|^{2} M_{g}\left(1+2 M_{a}\right)} \int_{0}^{t} p(s) \psi(v(s)) d s \\
\leq & k_{1}+k_{2} \int_{0}^{t} \frac{v(s)}{(t-s)^{1-\beta}} d s+k_{3} \int_{0}^{t} p(s) \psi(v(s)) d s .
\end{aligned}
$$

By Lemma 2.4, we get

$$
v(t) \leq B_{0}\left(k_{1}+k_{3} \int_{0}^{t} p(s) \psi(v(s)) d s\right),
$$

where

$$
B_{0}=e^{k_{2}^{n} \Gamma(\beta)^{n} b^{n \beta} / \Gamma(n \beta)} \sum_{j=1}^{n-1}\left(\frac{k_{2} b^{\beta}}{\beta}\right)^{j} .
$$

Let us take the right-hand side of the above inequality as $\mu(t)$. Then $\mu(0)=B_{0} k_{1}, v(t) \leq$ $\mu(t), t \in J$ and

$$
\mu^{\prime}(t) \leq B_{0} k_{3} p(t) \psi(v(t))
$$

Since $\psi$ is nondecreasing, we have

$$
\begin{aligned}
\mu^{\prime}(t) & \leq B_{0} k_{3} p(t) \psi(\mu(t)) \\
& =\bar{\mu}(t) \psi(\mu(t)) .
\end{aligned}
$$


It follows that

$$
\begin{aligned}
\int_{\mu(0)}^{\mu(t)} \frac{1}{\psi(s)} d s & \leq \int_{0}^{b} \overline{\mu(s)} d s \\
& \leq \int_{B_{0} K_{1}}^{\infty} \frac{1}{\psi(s)} d s
\end{aligned}
$$

which indicates that $\mu(t)<\infty$. Thus, there exists a constant $K$ such that $\mu(t) \leq K, t \in J$. Furthermore, we see that $\left\|y_{t}+\tilde{\phi}_{t}\right\|_{\mathcal{B}_{h}}^{2} \leq v(t) \leq \mu(t) \leq K, t \in J$.

Theorem 3.1 Assume that the assumptions (H1)-(H6) hold. The problem (1.1)-(1.3) has at least one mild solution on $J$.

Proof Let us take the set

$$
G(\Phi)=\left\{x \in \mathcal{B}_{h}: x \in \lambda \Phi(x) \text { for some } \lambda \in(0,1)\right\}
$$

Then for any $x \in G(\Phi)$, we have

$$
\left\|x_{t}\right\|_{\mathcal{B}_{h}}^{2}=\left\|y_{t}+\tilde{\phi}_{t}\right\|_{\mathcal{B}_{h}}^{2} \leq K, \quad t \in J
$$

where $K>0$ is a constant in Lemma 3.2. This show that $G$ is bounded on $J$. Hence from Theorem 2.1 there exists a fixed point $x(t)$ for $\Phi$ on $\mathcal{B}_{h}$, which is a mild solution of (1.1)-(1.3) on $J$.

\section{An example}

As an application of Theorem 3.1, we consider the impulsive neutral stochastic functional integro-differential inclusion of the following form:

$$
\begin{aligned}
& \frac{\partial}{\partial t}\left(z(t, x)+g\left(t, z(t-h, x), \int_{0}^{t} a(t, s, z(s-h, x)) d s\right)\right) \\
& \quad \in \frac{\partial^{2}}{\partial x^{2}} z(t, x)+\left(f(t, z(t-h, x))+\left[Q_{1}(t, z(t-h, x)), Q_{2}(t, z(t-h, x))\right]\right) d w(t), \\
& \quad 0 \leq x \leq \pi, t \in J, t \neq t_{k}, \\
& \Delta z\left(t_{k}, x\right)=z\left(t_{k}^{+}, x\right)-z\left(t_{k}^{-}, x\right)=I_{k}\left(z\left(t_{k}^{-}, x\right)\right), \quad k=1,2, \ldots, m, \\
& z(t, 0)=z(t, \pi)=0, \quad t \in J, \\
& z(t, x)=\rho(t, x), \quad-\infty<t \leq 0,0 \leq x \leq \pi,
\end{aligned}
$$

where $J=[0, b], k=1,2, \ldots, m, z\left(t_{k}^{+}, x\right)=\lim _{h \rightarrow 0^{+}} z\left(t_{k}+h, x\right), z\left(t_{k}^{-}, x\right)=\lim _{h \rightarrow 0^{-}} z\left(t_{k}+h, x\right)$, $Q_{1}, Q_{2}: J \times \mathbb{R} \rightarrow \mathbb{R}$ are two given functions and $w(t)$ is a one-dimensional standard Wiener process. We assume that for each $t \in J, Q_{1}(t, \cdot)$ is lower semicontinuous and $Q_{2}(t, \cdot)$ is upper semicontinuous. Let $J_{1}=(-\infty, b]$ and $H=L^{2}([0, \pi])$ with norm $\|\cdot\|$. Define $A: H \rightarrow$ $H$ by $A v=v^{\prime \prime}$ with domain $D(A)=\left\{v \in H: v, v^{\prime}\right.$ are absolutely continuous, $v^{\prime \prime} \in H, v(0)=$ $v(\pi)=0\}$. Then

$$
A v=\sum_{n=1}^{\infty} n^{2}\left(v, v_{n}\right), \quad v \in D(A)
$$


where $v_{n}=\sqrt{\frac{2}{\pi}} \sin (n s), n=1,2, \ldots$, is the orthogonal set of eigenvectors in $A$. It is well known that $A$ is the infinitesimal generator of an analytic semigroup $S(t), t \geq 0$ in $H$ given by

$$
S(t) v=\sum_{n=1}^{\infty} e^{-n^{2} t}\left(v, v_{n}\right) v_{n}, \quad v \in H
$$

For every $v \in H,(-A)^{-\frac{1}{2}} v=\sum_{n=1}^{\infty} \frac{1}{n}\left(v, v_{n}\right) v_{n}$ and $\left\|(-A)^{-\frac{1}{2}}\right\|=1$. The operator $(-A)^{\frac{1}{2}}$ is given by

$$
(-A)^{\frac{1}{2}} v=\sum_{n=1}^{\infty} n\left(v, v_{n}\right) v_{n}
$$

on the space $D\left((-A)^{-\frac{1}{2}}\right)=\left\{v \in H: \sum_{n=1}^{\infty} n\left(v, v_{n}\right) v_{n} \in H\right\}$. Since the analytic semigroup $S(t)$ is compact [10], there exists a constant $M>0$ such that $\|S(t)\| \leq M$ and satisfies (H1). Now, we give a special $\mathcal{B}_{h}$-space. Let $h(s)=e^{2 s}, s<0$. Then $l=\int_{-\infty}^{0} h(s) d s=\frac{1}{2}$ and let

$$
\|\varphi\|_{\mathcal{B}_{h}}=\int_{-\infty}^{0} h(s) \sup _{s \leq \theta \leq 0}\left(E|\varphi(\theta)|^{2}\right)^{\frac{1}{2}} d s
$$

It follows from [5] that $\left(\mathcal{B}_{h},\|\cdot\|_{\mathcal{B}_{h}}\right)$ is a Banach space. Hence for $(t, \phi) \in[0, b] \times \mathcal{B}_{h}$, let

$$
\begin{aligned}
& \phi(\theta) x=\phi(\theta, x), \quad(\theta, x) \in(-\infty, 0] \times[0, \pi], \\
& z(t)(x)=z(t, x)
\end{aligned}
$$

and

$$
F(t, \phi)(x)=\left[Q_{1}(t, \phi(\theta, x)), Q_{2}(t, \phi(\theta, x))\right], \quad-\infty<\theta \leq 0, x \in[0, \pi] .
$$

Then (4.1)-(4.4) can be rewritten as the abstract form as the system (1.1)-(1.3). If we assume that $(\mathrm{H} 2)-(\mathrm{H} 6)$ are satisfied, then the system (4.1)-(4.4) has a mild solution on $[0, b]$.

\section{Competing interests}

The authors declare that they have no competing interests.

\section{Authors' contributions}

All authors contributed equally and significantly in writing this article. All authors read and approved the final manuscript.

\section{Author details}

'Department of Mathematics, Busan National Universeity, Busan, 609-735, South Korea. ${ }^{2}$ Department of Mathematics, Dongeui University, Busan, 614-714, South Korea.

Received: 11 September 2013 Accepted: 17 December 2013 Published: 15 Jan 2014

\section{References}

1. Chang, YK, Anguraj, A, Arjunan, MM: Existence results for impulsive neutral functional differential equations with infinite delay. Nonlinear Anal. Hybrid Syst. 2, 209-218 (2008)

2. Park, JY, Balachandran, K, Annapoorani, N: Existence results for impulsive neutral functional integro-differential equations with infinite delay. Nonlinear Anal. 71, 3152-3162 (2009)

3. Lin, $A, H u$, L: Existence results for impulsive neutral stochastic functional integro-differential inclusions with nonlocal initial conditions. Comput. Math. Appl. 59, 64-73 (2010)

4. $\mathrm{Hu}, \mathrm{L}$, Ren, Y: Existence results for impulsive neutral stochastic functional integro-differential equations with infinite delays. Acta Appl. Math. 111, 303-317 (2010) 
5. Pazy, A: Semigroups of Linear Operators and Applications to Partial Differential Equations. Applied Mathematical Sciences, vol. 44. Springer, New York (1983)

6. Hino, Y, Murakami, S, Naito, T: Functional Differential Equations with Infinite Delay. Lecture Notes in Mathematics, vol. 1473. Springer, Berlin (1991)

7. Chang, YK: Controllability of impulsive functional differential systems with infinite delay in Banach spaces. Chaos Solitons Fractals 33, 1601-1609 (2007)

8. Losta, A, Opial, Z: Application of the Kakutani-Ky-Fan theorem in the theory of ordinary differential equations or noncompact acyclic-valued map. Bull. Acad. Pol. Sci., Sér. Sci. Math. Astron. Phys. 13, 781-786 (1965)

9. Dhage, BC: Multi-valued mappings and fixed points I. Nonlinear Funct. Anal. Appl. 10, 359-378 (2005)

10. Hernandez, E: Existence results for partial neutral functional integro-differential equations with unbounded delay. J. Math. Anal. Appl. 292, 194-210 (2004)

10.1186/1687-1847-2014-17

Cite this article as: Park and Jeong: Existence results for impulsive neutral stochastic functional integro-differential inclusions with infinite delays. Advances in Difference Equations 2014, 2014:17

\section{Submit your manuscript to a SpringerOpen ${ }^{\circ}$ journal and benefit from:}

- Convenient online submission

- Rigorous peer review

- Immediate publication on acceptance

- Open access: articles freely available online

- High visibility within the field

- Retaining the copyright to your article 